Baltic Astronomy, vol. 20, 419-426, 2011

\title{
THE STARBURST - AGN CONNECTION: A CRITICAL REVIEW
}

\author{
P. Rafanelli ${ }^{1}$, G. La Mura ${ }^{1}$, D. Bindoni ${ }^{1}$, S. Ciroi ${ }^{1}$, V. Cracco ${ }^{1}$, F. Di Mille ${ }^{2}$ \\ and L. Vaona ${ }^{1}$ \\ 1 Dipartimento di Astronomia, Università di Padova, Vicolo dell'Osservatorio 3 , \\ Padova I-35122, Italy; piero.rafanelli@unipd.it \\ 2 Carnegie Institution of Washington, The Carnegie Observatories, 813 Santa \\ Barbara Street, Pasadena, California 91101, U.S.A.; fdimille@gmto.org
}

Received: 2011 August 8; accepted: 2011 August 15

\begin{abstract}
Nuclear activity and star formation processes represent two key elements in the evolution of galaxies across the cosmic ages. In spite of very different physical backgrounds, several arguments suggest that they should be closely connected. On the basis of simple theoretical considerations, the transport of appreciable amounts of fuel to the AGN scale is very likely to trigger star formation in the gas. Young stellar populations, in their turn, are expected to affect the properties of the interstellar medium, leading to a complex balance of interactions among nuclear activity and star formation. This scenario is also supported by the observation of super-massive black holes and host galaxy properties, which strongly suggest a common evolutionary track. However, despite several years of extensive investigation, the relationship among the two processes still has to be properly explained. Here we provide a review of some of the most important observations, which are relevant to the issue of the connection among AGN and starburst events. Based on a wide sample of observations, we present an analysis of the spectral signatures connected with AGN and star formation activity. Expanding the concept of the distinction among star forming galaxies and the true active nuclei, we provide systematic evidence for a role of recent starburst events in the circum-nuclear regions of active galaxies and discuss the possibility of its influence onto the AGN environment. We also analyze the age, mass and metallicity properties of star-forming and active galaxies, illustrating that they are arranged in a sequence that is consistent with the identified relation.
\end{abstract}

Key words: galaxies: active, star formation, stellar content

\section{INTRODUCTION}

Many nearby galaxies show some degree of ionization in the gaseous components of their nuclear and circum-nuclear regions, within $1 \mathrm{kpc}$ from the center. The source of this ionization can be fundamentally ascribed to two different types of activity: nuclear starburst events and non-thermal processes. In the first case, the sources of gas ionization are the massive OB stars, which provide a strong UV continuum in the first evolutionary stages of a very young stellar population. In 
the second, instead, the high energy continuum is provided by the accretion processes involving very hot gas flows in a so-called Active Galactic Nucleus (AGN) (e.g., see Rees 1984). According to a recent estimate of the spectral properties in the nuclear regions of nearby galaxies, it has been suggested that approximately $10 \%$ of galaxies show nuclear starburst activity (SBN). Similarly, $10 \%$ of galaxies host some kind of AGN, while the remainder has little or no evidence of particular processes.

In spite of different forms of nuclear activity, there is wide agreement on the assumption of the Unified Model for the interpretation of AGNs. Through the identification of the central engine with the gravitational field of a Super-Massive Black Hole $\left(\mathrm{SMBH}, 10^{7} M_{\odot} \leq M_{\mathrm{BH}} \leq 10^{9} M_{\odot}\right)$, indeed, the observed luminosities of up to $10^{46} \mathrm{erg} \mathrm{s}^{-1}$ can be achieved with the accretion of $(0.2-2) M_{\odot} \mathrm{yr}^{-1}$. The presence of an obscuring structure of dusty molecular gas, which may hide some details of the nuclear source, provides an explanation for the variety of observational features.

A large number of studies were focused onto the problem of star formation close to AGNs, mainly taking into account Seyfert 2 galaxies. The overall conclusion was that nuclear activity is associated with recent star formation (less than 100 Myr) in at least $30-50 \%$ of cases. This circumstance raised the problem of whether the two processes are physically related, a question that is known as a Starburst AGN connection. Among the many possible interpretations, there are authors who suggested that the SMBH may grow from the remnants of starburst (Weedman 1983; Norman \& Scoville 1988; Taniguchi et al. 1999; Ebisuzaki et al. 2001; Mouri \& Taniguchi 2002a), that it could be fed with material ejected by massive stars, that the starburst may mimic an AGN (e.g., Scoville \& Norman 1988), or that it might represent an evolutionary stage in a sequence of activity (Heckman et al. 1989; Mouri \& Taniguchi 1992, 2002b; Cid Fernandes et al. 2001; Storchi-Bergman et al. 2001).

Recently, several works dealt with gaseous dynamics and starburst activity in the nuclear region of active galaxies (see Ballantyne 2008; Vollmer et al. 2008; Popović et al. 2009; Wild et al. 2010; Hopkins \& Quataert 2010; Tadhunter et al. 2011). In particular, Davies et al. (2007) performed a direct investigation of the circum-nuclear regions of active galaxies, using high resolution integral field spectroscopy in the near IR. They found an overwhelming evidence of intensive star formation processes, which anticipated the onset of nuclear activity by a time scale that is consistent with the theoretical expectations required to accrete mass lost by stars onto the SMBH. On the basis of these results, here we discuss the properties of a large sample of low redshift galaxies, considering the possibility that an evolutionary sequence may connect star formation and nuclear activity in galaxies.

\section{SAMPLE SELECTION AND DESCRIPTION}

To investigate the relationship among star-forming galaxies (SFG) and AGNs, we started from the selection of a composite sample of normal and emission-line galaxies from the Sloan Digital Sky Survey Data Release 7 (SDSS DR7, Abazajian et al. 2009). Looking at the spectra of galaxies with redshifts $z \leq 0.1$ and with detected emission lines of [O III $] \lambda 5007,[\mathrm{O}$ II $] \lambda \lambda 3727,3729$ and $[\overline{\mathrm{O}} \mathrm{I}] \lambda 6300$, and requiring a $S / N \geq 3$ for [O I], we extracted 119226 spectra, that subsequently were divided into SFGs and AGNs by means of the Kewley et al. (2006) parameteriza- 

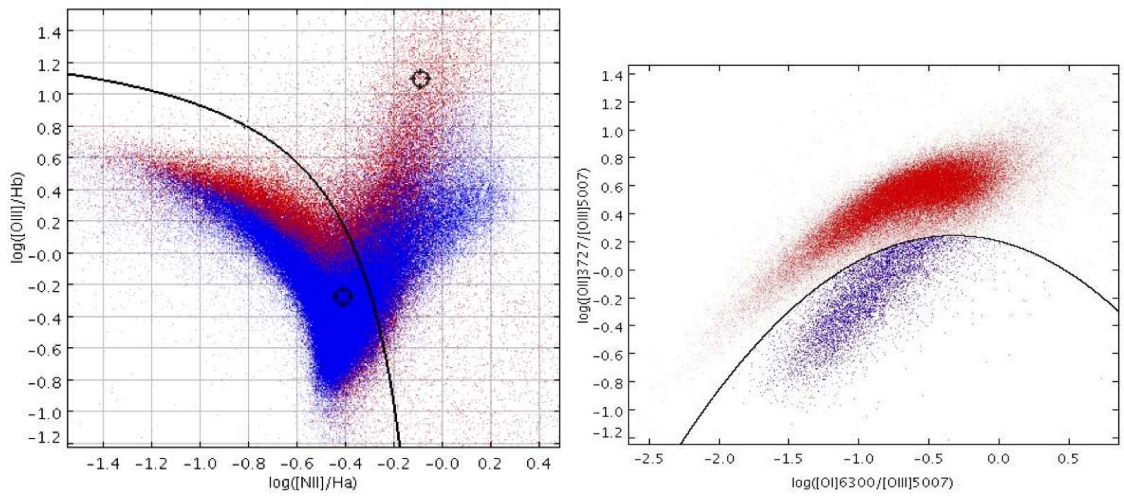

Fig. 1. Left panel. VO diagram for the whole emission-line sample, plotted before (blue) and after (red) correction for stellar contribution. The two circles mark the effect of correction onto a single object, taken as an example. Right panel. Diagnostic diagram for the identification of the SFG (red) and AGN (blue) branches according to Equation (1).

tion of the Veilleux-Osterbrock diagnostic diagrams. To account for the absorption components, which can considerably affect the intensity of the recombination lines used in these diagrams, we first modeled the stellar contribution to the spectra, which led to a modified distribution of the sample, that is illustrated in Figure 1. AGNs were subsequently identified as the sources satisfying the empirical relation (Vaona et al. in prep.):

$$
\begin{gathered}
\log ([\mathrm{O} \mathrm{II}] 3727 /[\mathrm{O} \mathrm{III}] 5007)<0.196-0.252 \log ([\mathrm{O} \mathrm{I}] 6300 /[\mathrm{O} \mathrm{III}] 5007)- \\
0.391 \log ([\mathrm{O} \mathrm{I}] 6300 /[\mathrm{O} \mathrm{III}] 5007)^{2}
\end{gathered}
$$

Restricting the sample to a redshift range of $0.04 \leq z \leq 0.08$, in order to cover a region of $2.4-4.8 \mathrm{kpc}$ around the nucleus in the fixed spectrograph fiber aperture and selecting only Seyfert 2 galaxies (S2G), we finally collected 1996 objects. A corresponding set of 1302 SFG was identified within the same region, by applying the diagnostic constraints (Rafanelli et al. 2009):

$$
\begin{aligned}
& \log ([\mathrm{O} \mathrm{III}] / \mathrm{H} \beta)<0.61 /[\log ([\mathrm{N} \mathrm{II}] / \mathrm{H} \alpha)]+1.3 \\
& \log ([\mathrm{O} \mathrm{III}] / \mathrm{H} \beta)<0.72 /[\log ([\mathrm{S} \mathrm{II}] / \mathrm{H} \alpha)]+1.3 \\
& \log ([\mathrm{O} \mathrm{III}] / \mathrm{H} \beta)<0.73 /[\log ([\mathrm{O} \mathrm{I}] / \mathrm{H} \alpha)]+1.33
\end{aligned}
$$

and requiring the $S / N>10$ in the continuum at $5500 \AA$. An additional set of 2000 normal galaxy spectra was also considered.

\section{DATA ANALYSIS}

\subsection{Stellar continuum}

In order to account for the stellar contributions, the spectra of all the selected galaxies were analyzed with the STARLIGHT code (Cid Fernandes et al. 2005), 


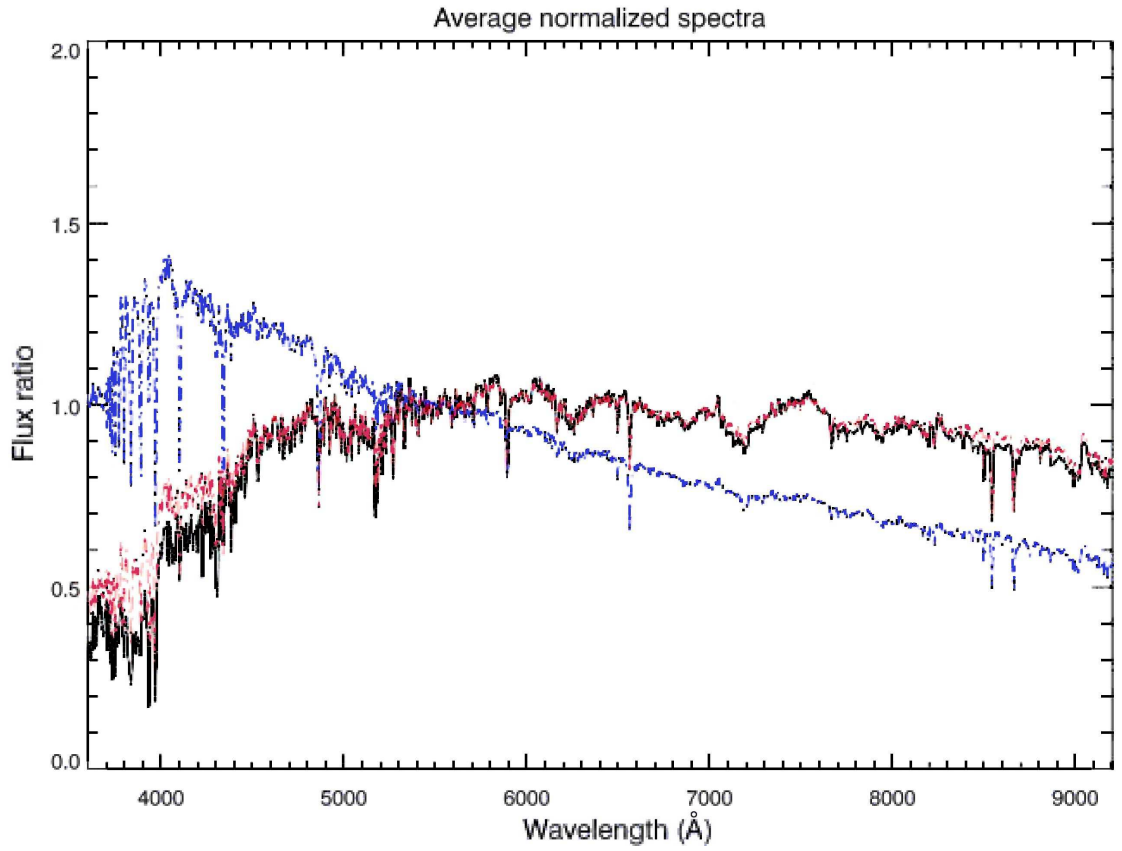

Fig. 2. Average spectra of the stellar components identified in SFGs (blue line), S2Gs (red) and normal galaxies (black).

which fits the observations using a linear combination of simple stellar populations, having the same age and a homogeneous chemical composition. The stellar population models were computed using a combination of 92 synthetic spectra, extracted from the library of Bruzual \& Charlot (2003) and sampled at the same spectral resolution of the SDSS. The models included simple stellar populations (SSP) with 23 different ages, in the range from $10^{6} \mathrm{yr}$ up to $1.3 \cdot 10^{10} \mathrm{yr}$, and four metallicities $\left(Z=0.2,0.4,1\right.$ and $\left.2.5 \times Z_{\odot}\right)$.

The average contributions of the stellar populations to the spectra of different object classes are illustrated in Figure 2. According to the expectations, the spectra of SFGs are significantly different, with respect to the other classes, due to the presence of young hot stars, which dominate the emission, yielding a blue peaked spectral energy distribution (SED). The spectra of normal galaxies and S2Gs, instead, are remarkably similar and characteristic of an evolved stellar population. In spite of the comparable SEDs, however, S2Gs show an average $4000 \AA$ break, which is clearly shallower than the corresponding feature of normal galaxies, indicating that an excess of hot stars of spectral class A populates the circum-nuclear region of active galaxies.

\subsection{The circum-nuclear environment}

The properties we identified as average trends actually mark an important distinction in the environment of galaxies, whose nuclei are affected by different physical processes. To investigate the details of such differences, we compared 
the features of the nuclear environment, looking both at the stellar and gaseous components of line emitting galaxies. Applying a grid of photoionization models to fit the spectra of S2Gs (see Rafanelli et al. 2008, 2010 for more details) and using the spectral indices proposed by Pilyugin \& Thuan (2005) for SFGs, we estimated the gas phase metallicities of our sample. This property, that tracks the evolution of the interstellar medium (ISM), as it is polluted by feedback from massive stars, was analyzed with respect to the characteristics of the underlying stellar component.
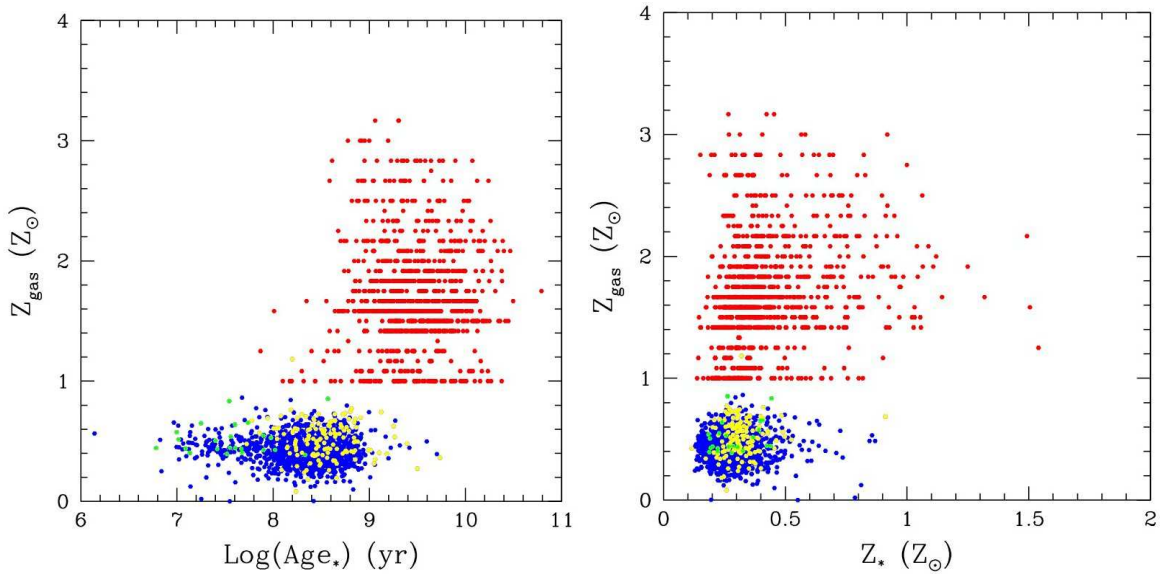

Fig. 3. Left panel. Gas phase metallicity compared to the age of stellar populations in starburst galaxies (green), low mass SFGs (blue), high mass SFGs (yellow) and S2Gs (red). Right panel. The same as in the left panel, but for a comparison with the stellar metallicities.
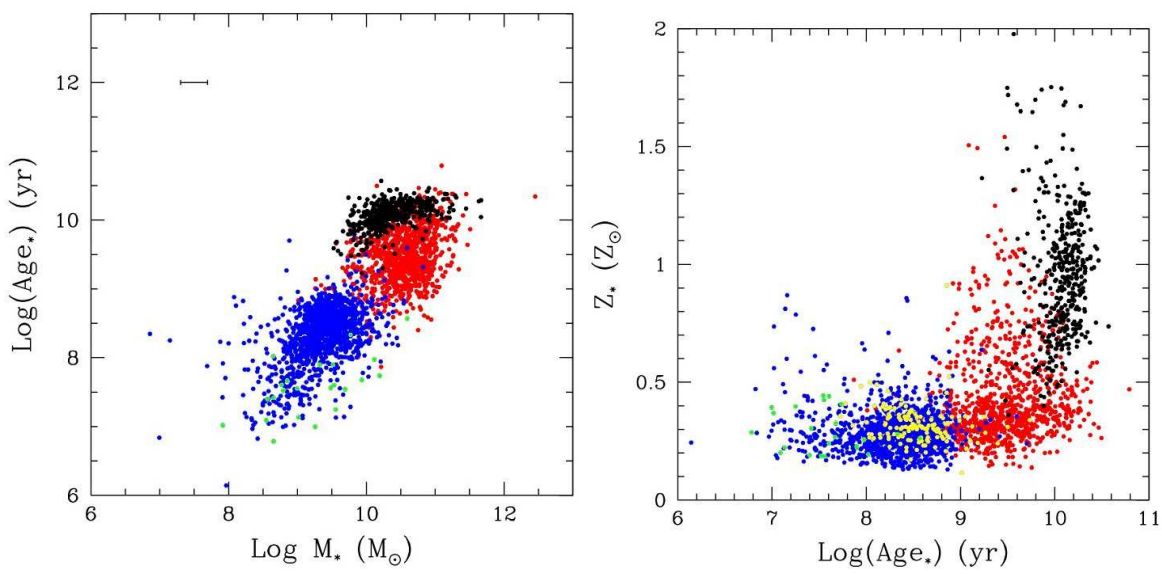

Fig. 4. Left panel. Relationship between the nuclear stellar population ages and total stellar masses of starburst galaxies (green), SFGs (blue), S2Gs (red) and normal galaxies (black). Right panel. Relationship between the stellar metallicity and age in the nuclear regions of starburst galaxies (green), low mass SFGs (blue), high-mass SFGs (yellow), S2Gs (red) and normal galaxies (black). 

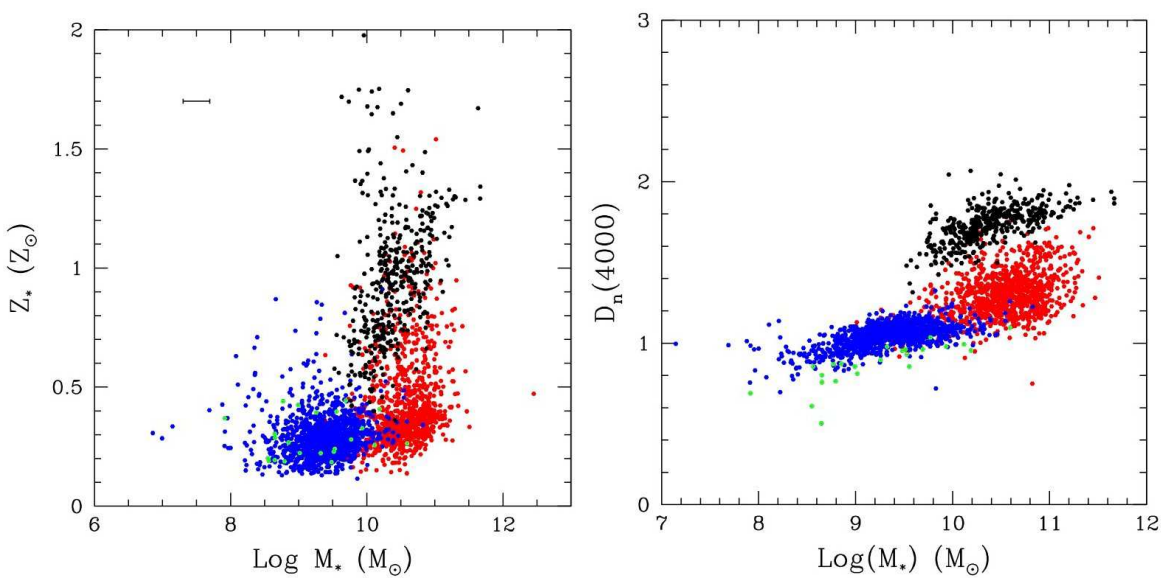

Fig. 5. The dependence of nuclear stellar population metallicity (left) and the 4000 $\AA$ break (right) on the stellar mass of the whole galaxies measured in the samples of starburst objects (green), SFGs (blue), S2Gs (red) and normal galaxies (black).

As it is shown in Figure 3, the gas of S2Gs appears as an evolved medium, with respect to the ISM we observe in SFGs. It can be noticed that the corresponding stellar populations show an overlapping sequence of increasing age, as we move from starburst objects (having $L_{\mathrm{H} \beta} \geq 10^{41} \mathrm{erg} \mathrm{s}^{-1}$ ) to more massive SFGs and S2Gs. Despite the different chemical compositions of the gas, however, the metallicity detected in the stellar population is mostly similar, with a slight over-abundance of heavy elements in S2Gs. This result indicates that both stellar populations belong to the same generation of stars, although the $\mathrm{O}-\mathrm{B}$ spectral class objects evolved off the main sequence in the case of S2Gs. In this interpretation it is likely that the gas ionization by the radiation produced in AGN becomes relevant a few Myr after a significant star formation activity occurred in the nucleus.

\subsection{Global properties}

A strong argument supporting the importance of a starburst - AGN connection resides in the several relationships which involve the SMBH mass and some fundamental properties of its host galaxy. Since the SDSS project provides, together with a wide spectroscopic archive, a homogeneous database of multiple band photometric observations, it has been possible to study the situation of the nuclear environment, observed in the spectra, with respect to the global structure of the host galaxies. In particular, Kauffmann et al. (2003) developed a useful technique to estimate the stellar mass of galaxies, using the observed luminosities and colors, to derive the appropriate mass-luminosity ratios.

Applying their results to our sample, we found that the starburst and SFGs are typically associated with low stellar mass objects, having a chemically unevolved ISM, while S2Gs show larger masses and evolved gas. As we illustrate in Figure 4, the mean age of the stellar populations increases from SFGs to S2Gs, while the normal galaxies are characterized by an old stellar population, distributed over the same range of masses covered by S2Gs. Most of the SFGs and S2Gs show a 
similar stellar metallicity (with only a weak dependence on the stellar mass of the whole galaxy), though the distribution of S2Gs also includes a high metallicity tail, which smoothly enters to the locus of normal galaxies. The samples of SFGs and S2Gs overlap in the mass range of $9.8 \leq \log \left(M_{*} / M_{\odot}\right) \leq 10.3$, similar to the typical scales of Luminous Infrared Galaxies. All these hints suggest that the process of circum-nuclear star formation is more commonly detected in objects with smaller masses, while it grows older and older towards the most massive objects, stopping in the region where AGNs become dominating.

In Figure 5 the stellar masses detected in our sample are compared with the metallicity of stellar population and with the depth of the $4000 \AA$ break, which we averagely estimated from our previous spectroscopic analysis. These data show that star formation processes are delayed in the objects with small masses, but while the distribution of SFGs appears homogeneous, S2Gs and quiescent galaxies also feature indications of stellar populations originating from the evolved medium, which might be the remnant of a weaker star formation activity surviving during the AGN phase. However, the clear gap observed in the distribution of the spectral breaks among normal and emission-line galaxies implies that the last process has only a secondary importance, since SFGs and S2Gs lie on a well-defined sequence, that is sharply interrupted in the region of active galaxies, turning them into the quiescent ones, with the same stellar masses, once the activity is over.

\section{CONCLUSIONS}

The properties observed within a wide sample of galaxies, including normal objects, as well as those with star forming and nuclear activity, are consistent with the expectation that star formation and AGNs should be actually related in the evolution of galaxies. In our analysis we identified several properties of the selected galaxies, which may lead to a more precise investigation of the problem as a whole and ultimately to a clearer view of the general picture.

We can summarize our conclusions noting that there is a range of galaxy masses $\left(9.8 \leq \log \left(M_{*} / M_{\odot}\right) \leq 10.3\right)$ where SFGs and AGNs are characterized by circumnuclear stellar populations of approximately the same age and similar chemical abundances in the ISM. Objects in this range are the oldest SFGs and the youngest AGNs, in terms of stellar population, and the metallicities of stars in SFGs within this mass range are indistinguishable from those of lower mass objects, while there are S2Gs showing evidence of further evolution. The metallicity of these stars seems to be independent from the mass of the whole galaxy and from the age of the circum-nuclear stellar population as it is the case for the gaseous component. This suggests that we are observing different stages of the evolution of the same star formation process. The circum-nuclear regions of SFGs with mass $9.8 \leq$ $\log \left(M_{\star} / M_{\odot}\right) \leq 10.3$, which clearly are in a more advanced evolutionary stage than the lower mass SFGs, seem to be the most promising candidate precursors of AGNs, if there is any causal link between the two phenomena.

ACKNOWLEDGMENTS. We thank the LOC and SOC for kind invitation to present this lecture and for the careful organization of the whole conference, and the referee for commenting this work. 


\section{REFERENCES}

Abazajian K. N., Adelman-McCarthy J. K., Agueros M. A. et al. 2009, ApJS, 182,543

Ballantyne D. R. 2008, ApJ, 685, 787

Bruzual G., Charlot S. 2003, MNRAS, 344, 1000

Cid Fernandes R., Heckman T., Schmitt H. et al. 2001, ApJ, 558, 81

Cid Fernandes R., Mateus A., Sodré L. et al. 2005, MNRAS, 358, 363

Davies R. I., Müller S. F., Genzel R. et al. 2007, ApJ, 671, 1388

Ebisuzaki T., Makino J., Tsuru T. G. et al. 2001, ApJ, 562, L19

Heckman T. M., Blitz L., Wilson A. S. et al. 1989, ApJ, 342, 735

Hopkins P. F., Quataert E. 2010, MNRAS, 407, 1529

Kauffmann G., Heckman T. M., White S. D. M. et al. 2003, MNRAS, 341, 33

Kewley L. J., Groves B., Kauffmann G., Heckman T. 2006, MNRAS, 372, 961

Mouri H., Taniguchi Y. 1992, ApJ, 386, 68

Mouri H., Taniguchi Y. 2002a, ApJ, 566, L17

Mouri H., Taniguchi Y. 2002b, ApJ, 565, 786

Norman C., Scoville N. 1988, ApJ, 332, 124

Pilyugin L. S., Thuan T. X. 2005, ApJ, 631, 231

Popović L. Č., Smirnova A. A., Kovačević J. et al. 2009, AJ, 137, 3548

Rafanelli P., Vaona L., Ciroi S., Di Mille F. 2008, JPhCS, 133, 2026

Rafanelli P., D'Abrusco R., Ciroi S. et al. 2009, NewAR, 53, 186

Rafanelli P., Vaona L., D'Abrusco R. et al. 2010, MSAIS, 15, 75

Rees M. J. 1984, ARA\&A, 22, 471

Scoville N., Norman C. 1988, ApJ, 332, 163

Storchi-Bergmann T., González Delgado R. M., Schmitt H. R. et al. 2001, ApJ, 559,147

Tadhunter C., Holt J., González Delgado R. et al. 2011, MNRAS, 412, 960

Taniguchi Y., Ikeuchi S., Shioya Y. 1999, ApJ, 514, L65

Vaona L., Ciroi S., Cracco V., Rafanelli P. 2011, in prep.

Vollmer B., Beckert T., Davies R. I. 2008, A\&A, 491, 441

Weedman D. W. 1983, ApJ, 266, 479

Wild V., Heckman T., Charlot S. 2010, MNRAS, 405, 933 